\title{
Introduction to the HICSS-50 Minitrack on Practice-based IS Research
}

\author{
Dorothy E. Leidner \\ Baylor University \\ dorothy_leidner@baylor.edu \\ Ester Gonzalez \\ California State Fullerton \\ esgonzalez@exchange.fullterton.edu
}

\author{
Bill Kettinger \\ University of Memphis \\ wjkttngr@memphis.edu \\ Michael Milovich \\ Rowan University \\ milovich@rowan.edu
}

The debate over rigor and relevance has extended many decades with rigor leading the way in the overwhelming majority of our academic journals where theory contribution and methodological excellence transcend any practical implication the research may have. Practice-based research aspires to bridge the gap between academic theory and practice; it aspires both to introduce researchers to state of the art practices and issues from industry as well as introduce managers to research that makes sense of and brings coherence to the issues they face. The methods used in practice-based research are often exploratory, field-based studies involving interviews, observations, and/or descriptive surveys. The intense pressure to achieve methodological distinction and theoretical contribution often results in very current practice-based topics being eschewed by researchers, because the topics themselves are not mature enough in practice to achieve desirable samples or sample sizes, nor are they conducive to theorizing since so little is known. These are precisely the reasons that exploratory, practice-based research can play a tremendous role in helping establish and lay the foundations of a research domain while providing insights into an emerging topic.

This year, our minitrack features one session with 3 papers. The first presentation will be based on the paper "Hunch Mining: Intuition Augmented with Cognitive Computing, Analytics, and Artificial Intelligence," by H. James Nelson, Terry Clark, and Ian Stewart. This fascinating paper introduces the notion of hunch mining to describe how latent intuitions, or hunches, from corporate decision makers as well as workers can be used as models for data analytics. The authors develop an Organizational Hunch Matrix and show how cognitive computing engineering can draw on different data sources for hunches and incorporate these into data analytics. The authors of this paper represent both academia (Southern Illinois
University) and industry (Presence Health), underscoring the tight linked between academia and industry that is encouraged by practice-based research.

The second presentation is based on the work of Hanna Timonen, from Aalto University in Finland and Johanna Vuori from the Haaga-Helia University of Applied Sciences in Finland. Their paper "Visibility of Work: How Digitalization Changes the Workplace" describes how digitalization changes the nature of work in the context of business-to-business sales work. The authors investigated the business-tobusiness sales force in a small SaaS company that was in the midst of adopting a new digital sales model. In particular, they examined how the digital sales model changed work visibility both in relation to co-works and in relation to customers. The case study shows how the B2B sales work increased in visibility in relation to co-workers but decreased in relation to customers. This resulted in changes to the selling styles of sales reps to a more consultative role. The authors offer important theoretical and managerial implications.

The third presentation is based on the paper, "An Exploratory Study of Current Information Security Training and Awareness Practices in Organizations" by Moneer Alshaiskh, Sean Maynard, Atif Ahmad and Shanton Chang all from the University of Melbourne. This study reports the findings from an exploratory field study of semi-structured interviews with senior and middle level IS security managers. The paper examines four central themes related to how an IS training and awareness program is implemented in organizations. The four areas concern the approach itself (ad-hoc vs. formal), the content of such programs (knowledge, motivation, and attitude), the competition for employees' attention, and the issues related measuring the effectiveness of the ISTA program. The authors provide guidelines to IS security managers developing security awareness and training programs. 